\title{
A mechanisms-based model for dynamic behavior and fracture of geomaterials
}

\author{
A. Zubelewicz ${ }^{1 *}$, E. Rougier ${ }^{1}$, M. Ostoja-Starzewski ${ }^{2}$, E.E. Knight ${ }^{1}$, C. Bradley ${ }^{1}$, and \\ H.S. Viswanathan ${ }^{1}$ \\ ${ }^{1}$ Los Alamos National Laboratory, NM, USA \\ ${ }^{3}$ Dept. of Mechanical Science and Engineering, University of Illinois at Urbana- \\ Champaign, Urbana, IL, USA \\ *corresponding author \\ Alek \& Research Associates, LLC, Los Alamos, NM, 87544, USA \\ Alek.Zubelewicz@gmail.com
}

\begin{abstract}
A mechanisms-based fracture model applicable to a broad class of earth and earth-like materials is presented. The key features the model captures are: (1) material anisotropy; (2) rate-sensitive directional fracture; (3) dilatational friction; (4) dynamic overstress in loading extremes, where the rate of supplied energy is not fully compensated by the rate of the energy redistribution and release and, lastly, (5) spatial stochasticity due to material heterogeneity. In comparison with more traditional phenomenological descriptions, the contribution of the proposed approach is the utilization of tensor representation theory; the theory is suitable for converting observed deformation and fracture mechanisms into a precise mathematical description of the material's behavior.
\end{abstract}




\section{Introduction}

Despite notable successes, the development of a predictive fracture model for geomaterials still poses a challenge. A broad review of fracture mechanisms in rocks is presented in $[1,2]$. The most common approach in modeling brittle fracture relies on incorporating damage characteristics into the Gibbs free energy [3, 4, and 5]. Several constitutive models have been proposed, and among them the microstructural models discussed in [6-8] are worth noting. The microplane model $[9,10]$ is the closest to our approach and is based on the simple idea of tracking fracture at microplanes with predetermined orientations. In this setting, the fracture planes carry information about the damage process in a physically justifiable manner. Furthermore, an assumption made in the microplane model is that the macro-damages can be treated as weakly interacting cracks. Since the crack opening strain is collected from the individual micro-cracks, this description is suitable for predicting the material behavior at early stages of the damage process. The cracks are characterized in terms of the crack surface area, while their orientations define the relevant stress tractions. In other approaches, viscous-like stress intensity factors are incorporated into the material description [11]. The time dependence is shown to replicate observed rock strengthening in the Hopkinson Bar strain rate regime $\left(10^{3} / \mathrm{s}\right.$ to $\left.10^{4} / \mathrm{s}\right)$. However, it is not clear whether the relationship holds at extreme strain rates (shock conditions).

The proposed approach utilizes results obtained in [12, 13], where the tensor representation theory is shown to be a very useful tool in the hands of a modeler [14]. First, we determine the dominant deformation and fracture mechanisms in a geomaterial. The selected mechanisms are incorporated into the mechanisms-based visco-plasticity model. In geomaterials the material's strength is mildly strain rate dependent until the point when the rate of supplied energy becomes comparable with the rate of the energy redistribution and release due to cracking. At extreme conditions the balance is violated and the material has an excess of energy. We study the mechanism by which, instead of a single dominant crack, the dynamically overstressed heterogeneous material experiences complex fractures and, in this manner, efficiently converts the externally supplied energy into the crack surface formation energy. 


\section{Tensor representations}

In the proposed fracture model, fracture processes are described in terms of dyadic products $N_{i j}=n_{i} n_{j}$, where unit vector $n_{k}$ defines the crack orientation. We want the tensor to be expressed in terms of another symmetric tensor (for instance, stress). In here, we choose the base tensor to be $T_{k i}=T_{i k}$ and, then, express $N_{i j}$ in terms of $T_{k l}$ such that $N_{i j}^{T}=N_{i j}^{T}\left(T_{k l}\right)$. The tensor representation theory [14] says that any symmetric second order tensor can be represented by another symmetric tensor, if the original tensor and its representation have the same first, second and third invariants. From the Cayley-Hamilton theorem we write

$$
N_{i j}^{T}=\alpha_{0} \delta_{i j}+\alpha_{1} T_{i j}^{D}+\alpha_{2} T_{i k}^{D} T_{k j}^{D}
$$

where the deviator of $T_{k l}$ is $T_{k l}^{D}=T_{k l}-T_{i i} \delta_{k l} / 3$. There are three representations of the dyadic product each aligned with a principle orientation of $T_{k l}$. First, we notice that the tensor representation $N_{i j}^{T}$ taken to the first, second, and third power is still the same tensor $N_{i j}=N_{i k} N_{k j}=N_{i k} N_{k l} N_{l j}$ and their traces are equal to one. As a result, we find three solutions in terms of $\left\{\alpha_{0}, \alpha_{1}, \alpha_{2}\right\}$ and, consequently, we have three tensor representations. It can be shown that the eigenvector consists of $T_{1}=N_{i j}^{1}\left(\alpha_{0}^{1}, \alpha_{1}^{1} \alpha_{2}^{1}\right) T_{i j}$, $T_{2}=N_{i j}^{2}\left(\alpha_{0}^{2}, \alpha_{1}^{2} \alpha_{2}^{2}\right) T_{i j}$ and $T_{3}=N_{i j}^{3}\left(\alpha_{0}^{3}, \alpha_{1}^{3} \alpha_{2}^{3}\right) T_{i j}$. For instance, when tensor $T_{k l}$ is stress then the eigenvector consists of principal stresses $\left\{\sigma_{1}, \sigma_{2}, \sigma_{3}\right\}$, where $\sigma_{1}>\sigma_{2}>\sigma_{3}$. The first set of the alpha parameters is

$$
\begin{gathered}
\alpha_{0}^{1}=\frac{1}{3}\left(1-2 \cos \frac{\pi+\varphi}{3} \sec \varphi\right) \\
\alpha_{1}^{1}=\frac{8 \sqrt{3} \cos \frac{\varphi}{3}-2 \sqrt{3} \cos \varphi-3 \sqrt{3} \sec \varphi-6 \sin \varphi}{6 \sqrt{J_{2}}\left(2 \cos \frac{2 \varphi}{3}-1\right)\left(\sqrt{3}-2 \sin \frac{2 \varphi}{3}\right)} \\
\alpha_{2}^{1}=\frac{\cos \frac{\pi+\varphi}{3} \sec \varphi}{J_{2}}
\end{gathered}
$$

The second representation is described by 


$$
\begin{gathered}
\alpha_{0}^{2}=\frac{1}{3}\left(1+2 \cos \frac{\pi+\varphi}{3} \sec \varphi\right) \\
\alpha_{1}^{2}=\frac{2 \tan \varphi \cos \frac{\varphi^{2}}{3} \sec \varphi}{\sqrt{3 J_{2}}\left(1+2 \cos \frac{\varphi}{3} \sec \varphi\right)} \\
\alpha_{2}^{2}=-\frac{\cos \frac{\varphi}{3} \sec \varphi}{J_{2}}
\end{gathered}
$$

and the third one becomes

$$
\begin{gathered}
\alpha_{0}^{3}=\frac{1}{3}\left(1-2 \cos \frac{\pi-\varphi}{3} \sec \varphi\right) \\
\alpha_{1}^{3}=\frac{8 \sqrt{3} \cos \frac{\varphi}{3}+2 \sqrt{3} \cos \varphi-3 \sqrt{3} \sec \varphi-6 \sin \varphi}{6 \sqrt{J_{2}}\left(2 \cos \frac{2 \varphi}{3}-1\right)\left(\sqrt{3}-2 \sin \frac{2 \varphi}{3}\right)} \\
\alpha_{2}^{3}=\frac{\cos \frac{\pi-\varphi}{3} \sec \varphi}{J_{2}}
\end{gathered}
$$

In all the cases, the angle is $\varphi=\sin ^{-1} J_{3}\left(27 / 4 J_{2}^{3}\right)^{1 / 2}$, while the second and third invariants are $J_{2}=T_{i j}^{D} T_{i j}^{D} / 2$ and $J_{3}=T_{i k}^{D} T_{k l}^{D} T_{l i}^{D} / 3$, respectively.

\section{Friction-induced visco-plasticity}

Frictional plastic deformation consists of slippages along crack surfaces and there is an out-of-plane dilatation due to crack roughness. The shear strain rate is defined in terms of the Mises-Schleicher concept developed independently by von Mises and Schleicher [15]. In our case, the mechanism of the frictional shear flow is

$$
M_{i j}=\sqrt{3} \frac{s_{i j}}{\sqrt{J_{2}^{\sigma}}}+q\left(J_{2}^{\sigma}\right) \delta_{i j}
$$

such that

$$
\dot{\varepsilon}_{i j}^{p}=\frac{1}{2} M_{i j} \dot{e}_{e q}^{p}
$$

The flow tensor $M_{i j}$ is co-rotational with stress deviator $S_{i j}=\sigma_{i j}-\delta_{i j} \sigma_{k k} / 3$ and is a function of the second invariant of stress deviator $J_{2}^{\sigma}=S_{i j} S_{i j} / 2$. Also, it includes the dilatation part scaled through internal friction $q=q_{0} /\left[1+\left(\sqrt{3 J_{2}^{\sigma}} / \sigma_{q}\right)^{n_{q}}\right]$, where $q_{0}$ is 
friction parameter. Friction is affected by shear (second invariant of stress deviator), where $\sigma_{q}$ and $n_{q}$ are constants. The equivalent plastic strain rate $\dot{e}_{e q}^{p}$ is coupled with an equivalent stress. From the requirement of measure invariance (independence from the frame of description) of plastic power $\left(\sigma_{i j} \dot{\varepsilon}_{i j}^{p}=\sigma_{e q} \dot{e}_{e q}^{p}\right)$, the equivalent stress becomes $\sigma_{e q}^{p}=M_{i j} \sigma_{i j} / 2=\sqrt{3 J_{2}^{\sigma}}+3 p q / 2$. In the next step, we develop a constitutive relationship between the equivalent stress and strain rate. In here, the relationship is proposed in a pseudo-linear form

$$
\dot{e}_{e q}^{p}=\dot{\Lambda}_{p} \frac{\sigma_{e q}^{p}}{\sigma_{0}^{p}}
$$

where $\sigma_{0}^{p}$ is the material's strength consisting of static strength $\sigma_{0}^{S}$ and dynamic overstress $\sigma_{0}^{D}$ such that $\sigma_{0}^{p}=\left(1-\eta_{c}\right)\left(\sigma_{0}^{S}+\sigma_{0}^{D}\right)$. The role of the two strengths and damage $\left(\eta_{c}\right)$ are discussed later. We correct the linear strain rate dependence given by $\left(\sigma_{e q}^{p} / \sigma_{0}^{p}\right)$ and make it non-linear by using a rate-sensitive factor $\dot{\Lambda}_{p}=\dot{e}_{N}^{0}\left(\dot{e}_{N}^{t} / \dot{e}_{N}^{0}\right)^{\omega_{p}}$, where the parameter $\omega_{p}$ is a material constant. Lastly, we choose the scaling parameter $\dot{e}_{N}^{0}$ to be equal to $1 / s$. The normalized total strain rate is $\dot{e}_{N}^{t}=\sqrt{\dot{\varepsilon}_{i j}^{t} \dot{\varepsilon}_{i j}^{t}} / 2$. In this construction, we allow the effective stress exponent to vary throughout the deformation process. At an advanced stage of the plastic deformation, where $\dot{\varepsilon}_{i j}^{p} \rightarrow \dot{\varepsilon}_{i j}^{t}$ we have $\dot{e}_{e q}^{p} \propto\left(\sigma_{e q}^{p} / \sigma_{0}^{p}\right)^{1 /\left(1-\omega_{p}\right)}$; thereby assuring a smooth elasto-plastic transition. As geomaterials generally exhibit low strain rate dependence, the parameter $\omega_{p}$ is expected to have values only slightly smaller than one.

\section{Fracture tensor}

In geomaterials, the fracture processes occur in tension, compression and shear. Multiple tensile micro-cracks form on microplanes and are predominantly aligned with the direction of maximum tensile stress. The extent of the damage is correlated with the amount of work required for the micro-cracks to become fully opened [16]. In order to monitor the damage process, we construct a fracture tensor, whose rate is defined as follows

$$
\dot{\Omega}_{i j}=\dot{G}_{f} N_{i j}^{\sigma}
$$


where the tensor $N_{i j}^{\sigma}=n_{i} n_{j}$ is a dyadic product of vector $n_{i}$ aligned with the direction of maximum tensile stress. In section 2, we have expressions for the three representations $N_{i j}^{\sigma}\left(\sigma_{k l}\right)$ and in here we take the first one defined by parameters $\left\{\alpha_{0}^{1}, \alpha_{1}^{1}, \alpha_{2}^{1}\right\}$. Fracture energy is equal to the work needed for opening the cracks, thus $\dot{G}_{f}=\left(N_{i j}^{\sigma} \sigma_{i j}\right) \times\left(l_{c} N_{k l}^{\sigma} \dot{\varepsilon}_{k l}^{e}\right)$. The normal component of traction vector is $N_{i j}^{\sigma} \sigma_{i j}$ and the rate of crack opening displacements becomes $l_{c} N_{k l}^{\sigma} \dot{\varepsilon}_{k l}^{e}$. Lastly, the characteristic fracture length $l_{c}$ is correlated with the Stochastic Volume Element (SVE) of the material. This length and the stochasticity of fracture are discussed later. This energy is accumulated only during an active fracture process $\left(N_{i j}^{\sigma} \sigma_{i j}>0\right)$, where the rate of the crack opening displacements is a nonnegative quantity $\left(l_{c} N_{k l}^{\sigma} \dot{\varepsilon}_{k l}^{e}>0\right)$. The fracture tensor records history of damage $\Omega_{i j}=\int_{t_{0}}^{t} \dot{\Omega}_{i j}(s) d s$ incurred by the material starting from the instance $t_{0}$.

\section{Anisotropic elasticity with fracture}

We monitor progression of fracture in terms of three principle orientations of the tensor $\Omega_{i j}$. Damage in the primary orientation (dominant tensile fracture) is determined by the eigentensor $\mathrm{N}_{i j}^{\Omega-1}\left(\Omega_{k l}\right)$. There is also the secondary orientation $\mathrm{N}_{i j}^{\Omega-2}\left(\Omega_{k l}\right)$ and we include the possibility for the damage growth in the third orientation $\mathrm{N}_{i j}^{\Omega-3}\left(\Omega_{k l}\right)$. Note that the three orientations are orthogonal and, therefore, the tensors must satisfy the following relation $N_{i j}^{\Omega-1}+N_{i j}^{\Omega-2}+N_{i j}^{\Omega-3}=\delta_{i j}$. Consequently, the model captures fracture along any complex loading path. In addition, we include a rubble-like fracture in compression. The effective elastic properties evolve as the fracture process proceeds. We construct an elasticmatrix tensor, which we write in the following form

$$
\Delta_{i j}=\left(1-\eta_{c}\right)\left(\omega_{1} N_{i j}^{1}+\omega_{2} N_{i j}^{2}+\omega_{3} N_{i j}^{3}\right)-\left(\eta_{\Omega-1} N_{i j}^{\Omega-1}+\eta_{\Omega-2} \mathrm{~N}_{i j}^{\Omega-2}+\eta_{\Omega-3} \mathrm{~N}_{i j}^{\Omega-3}\right) .
$$

In this expression, we include the preexisting (natural) material's anisotropy. For simplicity, the geomaterial exhibits directional elastic properties in three directions $N_{i j}^{r}$ with the intensities defined by the parameters $\omega_{r}$, where $r=1,3$. The responses are scaled through the compressive damage $\eta_{c}$ due to friction $W^{p}=\int_{t_{0}}^{t} \sigma_{i j}(s) \dot{\varepsilon}_{i j}^{p}(s) d s$, which is 


$$
\eta_{c}=\frac{\left(\frac{W^{p}}{W^{0}}\right)^{m}}{1+\left(\frac{W^{p}}{W^{0}}\right)^{m}}
$$

In this expression, $W^{0}$ is critical friction dissipation and the exponent $m$ determines the rate of the damage accumulation. We carry information on fracture in the second part of the elastic-matrix tensor (9). The fracture intensities in the three principle orientations are defined through $\eta_{\Omega-1}, \eta_{\Omega-2}$ and $\eta_{\Omega-3}$. The amount of damage in each orientation is given by

$$
D_{r}=N_{i j}^{\Omega-r} \Omega_{i j}
$$

where $r=1,3$. Thus, the material may experience non-recoverable damage in the primary $(\Omega-1)$, secondary $(\Omega-2)$ and tertiary $(\Omega-3)$ orientations. However, the damage is accumulated only when the material is in tension $\left(D_{r}>0\right)$. The fracture functions are

$$
\eta_{\Omega-r}=\eta_{\Omega-r}^{\max } \frac{\left(\frac{D_{r}}{G_{f}^{0}}\right)^{n}}{1+\left(\frac{D_{r}}{G_{f}^{0}}\right)^{n}}
$$

As before, the exponent $n$ determines the rate of the fracture process. Since the material is anisotropic, the maximum allowable magnitude of tensile fracture is

$$
\eta_{\Omega-r}^{\max }=N_{i j}^{\Omega-r}\left(\omega_{1} N_{i j}^{1}+\omega_{2} N_{i j}^{2}+\omega_{3} N_{i j}^{3}\right)\left(1-\eta_{c}\right)
$$

We note that the isotropic damage in compression contributes to the damages in tension. The elastic-matrix tensor $\Delta_{i j}$ is now used in the material's elastic tensor

$$
C_{i j k l}=\lambda \Delta_{i j} \Delta_{k l}+\mu\left(\Delta_{i k} \Delta_{j l}+\Delta_{i l} \Delta_{j k}\right),
$$

where the average Lame constant is $\lambda$ and the average shear modulus is $\mu$. As usual, we assume strain rate additivity $\dot{\varepsilon}_{i j}^{t}=\dot{\varepsilon}_{i j}^{e}+\dot{\varepsilon}_{i j}^{p}$, where the rate of total strain consists of the rate of the elastic (reversible) strain $\dot{\varepsilon}_{i j}^{e}$ and the rate of dilatation friction $\dot{\varepsilon}_{i j}^{p}$. We accumulate elastic strain $\varepsilon_{i j}^{e}=\int_{t_{0}}^{t} \dot{\varepsilon}_{i j}^{e}(s) d s$ and, from there, we predict the components of the current 
stress, namely $\sigma_{i j}=C_{i j k l} \varepsilon_{k l}^{e}$. We should make a note that in the case of brittle materials the use of small strain theory is a justifiable approximation.

\section{Equation of state}

Geomaterials subjected to extreme dynamics are capable of forming shock waves. In our case, the pressure-volume equation of state (EOS) is based on a simplified MieGruneisen relationship, where we omit the contribution of temperature and express the relation in terms of the bulk modulus $B$ alone

$$
B=B_{0} \frac{1+\varepsilon_{k k}^{e}\left(1-\Gamma_{0} / 2\right)}{\left(1-S_{p} \varepsilon_{k k}^{e}\right)^{2}}
$$

In this formula, the reference bulk modulus is $B_{0}$ and the other two material parameters $\left(\Gamma_{0}\right.$ and $S_{p}$ ) are constants. The extreme allowable compressibility is $1 / S_{p}$. We emphasize that the EOS reflects the pressure-volume relation in a fracture-free elastic material.

\section{Dynamic overstress}

So far, we have described the mechanisms of dilatation slippages and fracture. Our next question is: What happens when the rate of the energy delivered to the material is comparable or exceeds the rate of the energy redistribution, dissipation and conversion? We study the dynamic problem under the assumption of plane stress and steady state, where the rate of the propagating cracks is included in the analysis. We skip the derivations because a similar problem has already been solved for metals, where the mechanism of the energy redistribution is linked to the mobility of dislocations $[17,18]$. In this case, the process is carried by nucleating and propagating cracks. Thus, we follow the derivations in [18] and find that the dynamic strengthening of the material in (7) can be expressed as $\sigma_{0}^{p}=\sigma_{0}^{S}+$ $\sigma_{0}^{D}$, where the static strength $\sigma_{0}^{S}$ is a constant and the formula for the dynamic overstress can be reduced to

$$
\sigma_{0}^{D}=\sigma_{0}^{S} \frac{\left(\frac{\dot{e}_{N}}{\dot{e}_{N}^{c r}}\right)^{2}}{1+\alpha_{D}-\left(\frac{\dot{e}_{N}}{\dot{e}_{N}^{c r}}\right)^{2}}
$$


where the normalized strain rate $\dot{e}_{N}$ is described earlier. The strain rate limit is defined in terms of the critical strain rate $\dot{e}_{N}^{c r}$, where $\dot{e}_{N} \leq \dot{e}_{N}^{c r}$, and the amount of the energy excess is controlled by the parameter $\alpha_{D}$.

\section{Mesh scalable stochasticity}

In geological materials, elastic properties are stochastic, as is the propensity for frictional slippages and the resistance to fracture. We assume that the resistance to tensile fracture $G_{f}^{0}$ has the dominant contribution to the sample-to-sample variability and, therefore, the fracture energy renders the geomaterial a random medium. In principle, by a random medium $\mathscr{R}$ we understand an ensemble of deterministic and deterministically responding heterogeneous specimens (or realizations) $R(\zeta)$ such that $\mathscr{R}=\{R(\zeta ; \zeta \in \mathscr{F})\}$. Each $R(\zeta)$ is a particular specimen seen in the laboratory [19]. All the specimens are nominally the same, but, in detail (i.e. on fine scales), they differ from one another. They are parameterized by sample events $\zeta$ belonging to the $\mathcal{F}$ space; the latter is well defined in spatial dimension with a probability measure $P$. Now, what distinguishes one $R(\zeta)$ from another is the spatial distribution of the fracture energy $G_{f}^{0}$. Thus, the random medium is specified through a scalar-valued random field $\mathscr{R}=\left\{G_{f}^{0}(\zeta, x, \delta) ; \zeta \in \mathcal{F}, x \in D\right\}$, where $D$ is the spatial domain of the body. In the above we also introduce a meso-scale $\delta=L / l_{c}$, which is a parameter specifying the scale of resolution (or measurement) of the $G_{f}^{0}$ property relative to the chosen micro-scale feature (such as grain size, characteristic sub-domain, etc.). It is expected that the finer the mesoscale, the stronger the random fluctuations of $G_{f}^{0}$ become. Moreover, we also assume that the fracture characteristics are statistically isotropic. Thus, $G_{f}^{0}$ is specified by its mean and the correlation function and the $d V$ volume (specified by the meso-scale $\delta$ of the random medium model) is the introduced earlier Statistical Volume Element. In most situations, we expect the scatter of the responses to decrease as the scale of resolution $\delta$ increases.

\section{Discussion}


The mechanisms-based model is implemented to a Lagrangian hydrocode developed at the Los Alamos National Laboratory. The code is called CASH. The fracture model is calibrated for Climax Stock granite for which experimental data in uniaxial tension, compression and stress triaxiality are available [20,21].

\subsection{Model calibration}

Material chosen in this analysis is granite (Climax Stock). The model is calibrated using material properties presented in [20] and [21]. For convenience, we collect material parameters in three groups; the first represents elastic constants, the second describes material's strength and the third defines fracture.

\subsubsection{Mass density and elastic properties}

Mass density and elastic constants $(\rho, B, \mu)$ for granite are given in [20]. EOS is defined in terms of $\left(\Gamma_{0}\right.$ and $\left.S_{p}\right)$ and the parameters are obtained from [22]. Climax Stock granite is considered an isotropic material and, therefore $\omega_{1}=\omega_{2}=\omega_{3}=1$.

\subsubsection{Strength}

Strength parameters include static strength $\sigma_{0}^{S}$ and internal friction $q$. The latter takes the following form $q=q_{0} /\left[1+\left(\sqrt{3 J_{2}^{\sigma}} / \sigma_{q}\right)^{n_{q}}\right]$ and is described in terms of coefficient $q_{0}$ and is affected by shear stress ratio $\left(\sqrt{3 J_{2}^{\sigma}} / \sigma_{q}\right)^{n_{q}}$ taken to the power $n_{q}$, where $\sigma_{q}$ and $n_{q}$ are constants. The parameters $\left(\sigma_{0}^{S}, q_{0}, \sigma_{q}, n_{q}\right)$ define stress envelope constructed in terms of Huber-von Mises stress and pressure (Fig. 9 of [21]):

$$
\sigma_{0}^{S}=\sigma_{e q}^{p}=\sqrt{3 J_{2}^{\sigma}}+3 p q / 2
$$

At low strain rates, the material's strain rate dependence is controlled by the parameter $\omega_{p}$. At very high rates, the increases of strength are much more pronounced. The dynamic overstress (12) used in (7) is calibrated with the use of critical strain rate $\dot{e}_{N}^{c r}$ and the overstress parameter $\alpha_{D}$. In geological materials, the critical strain rate should be in the range of hundreds per second, while the estimated overstress parameter is about 0.1 . 


\subsubsection{Fracture}

Tensile strength is estimated from uniaxial compression assuming that the ratio of the tensile and compressive strength is $1 / 10$. Damage parameters $\left(G_{f}^{0}\right.$ and $\left.n\right)$ affect both of the tensile strength and the slop of strain softening. Damage in compression is controlled by $\left(W^{0}\right.$ and $m$ ), where both the parameters are calibrated using the experimental data in [21]. All the material constants are collected in Table 1.

\subsection{Results}

Uniaxial stress-strain responses in compression and tension are shown in Figs. 1a and 1b. We have good agreement between the model predictions and the experimental data for uniaxial compression [20]. Unfortunately, the post-peak granite responses are not available. Note that damage occurs in compression and tension but the softening slope in tension is much steeper. The model correctly reproduces stress triaxiality data in [21] (Fig. 2). We test the model for granite subjected to strain-controlled cycling. The predictions are presented in Fig. 3. First, the material is severely fractured in tension (segment 1). Consequently, the unloading part of the process (segment 2) confirms a noticeable reduction of elastic stiffness. Subsequently, the material undergoes a recompression and, as shown in segment 3, elastic stiffness is significantly recovered. Next cycle causes unloading (segment 4) and brings the material to tension (segment 5). The follow up reloading closes tensile cracks (segment 6) and the material regains strength in compression (segment 7). The model captures the contribution overstress at high strain rates. As shown in Fig. 4, strength plotted as a function of strain rate follows the typical S-shape curve.

\section{Summary}

A mechanisms-based viscoplasticity model with dynamic overstress, anisotropy and fracture is developed for a general class of geomaterials. In this model, the selection of deformation and fracture mechanisms determines the shape of failure envelop and it dictates the rules of plastic flow. The compartmental construction of the model helps in an efficient calibration of material constants. We do not calibrate the contribution of fracture 
spatial stochasticity. This feature of the model is relevant but it can be tested only when solving boundary value problems.

\section{Acknowledgement}

This project has been performed under the auspices of the US Department of Energy. The Los Alamos National Laboratory is operated by Los Alamos National Security, LLC for the NNSA of the U.S. DOE under Contract No. DE-AC52-06NA25396. The authors wish to express their gratitude to Scott Broome of Sandia National Laboratories for providing experimental data for granite. The experimental study was sponsored by the SPE multi-institutional and interdisciplinary group of scientists and engineers from National Security Technologies (NSTec), Lawrence Livermore National Laboratory (LLNL), Los Alamos National Laboratory (LANL), Sandia National Laboratories (SNL), the Defense Threat Reduction Agency (DTRA), and the Air Force Technical Applications Center (AFTAC). 


\section{References}

1. Bieniawski ZT. Fracture dynamics of rock. Int. J. Fract. Mech. 1968;4:415-30.

2. Kranz RL. Microcracks in rocks: A review. Tectonophysics 1983;100:449-480.

3. Hill R, Rice JR. Elastic potentials and the structure of inelastic constitutive laws. SIAM J. Appl. Math. 1073;25:448-461.

4. Kachanov LM. A micro-crack model of rock inelasticity. Part I: Frictional sliding on micro-cracks and Part II: Propagation of micro-cracks. Mech. Mater. 1982;5:119-127.

5. Krajcinovic D. Damage Mechanics. Amsterdam: Elsevier; 1996.

6. Taylor LM, Chen EP, Kuszmaul JS. Microcrack-induced damage accumulation in brittle rock under dynamic loading. Comp. Meth. Appl. Mech. Eng. 1986;55:301-320.

7. Ashby MF, Sammis CG. The damage mechanics of brittle solids in compression. Pure Appl. Geophys. 1990; 133: 489-521.

8. Zuo QH, Dienes JK. On the stability of penny-shaped cracks with friction: the five types of brittle behavior. Int. J. Solids Struct. 2005;42:1309-26.

9. Bazant $\mathrm{ZP}, \mathrm{Oh} \mathrm{BH}$. Microplane model for progressive fracture of concrete and rock. ASCE J. Eng. Mech. 1985;111:559-582.

10. Bazant ZP, Prat PC. Microplane model for brittle-plastic material: I. Theory. ASCE J. Eng. Mech. 1988;114:1672-1688.

11. Bhat HS, Rosakis AJ, Sammis CG. A micromechanics based constitutive model for brittle failure at high strain rates, J. Appl. Mech. 2012;79: 031015.

12. Zubelewicz A. Overall stress and strain rates for crystalline and frictional materials. Int. J. Non-Linear Mech. 1990;25:389-393. 
13. Zubelewicz A, Ionita A, Ostoja-Starzewski M, et al. Fracture model for cemented aggregates. AIP Adv 2013; 3: 012119.

14. Zubelewicz A. Micromechanical study of ductile polycrystalline materials. J. Mech. Phys. Solids 1993;41: 1711-1722.

15. Hoffman O. The brittle strength of orthotropic materials, J. Compos. Maters. 1967;1: 200-205.

16. Zubelewicz A, Bazant ZP. Constitutive model with rotating active plane and true stresses. ASCE J. Eng. Mech. 1987;113:398-416.

17. Zubelewicz A. Metal behavior at extreme loading rates: Thermodynamics. Phys. Rev. B 2008;77: 214111.

18. Zubelewicz A. Metal behavior at extreme rates. Mech. Maters. 2009;41,:969-974.

19. Lee E, Finger M, Collins W. JWL equation of state coefficients for high explosives. Report UCID-16189, Lawrence Livermore National Laboratory, Livermore, California, 1973.

19. Ostoja-Starzewski M. Microstructural Randomness and Scaling in Mechanics of Materials. Boca Raton, Fla: CRC; 2008.

20. Broome S, Pfeifle T. Phase 1: Mechanical Property Test Results for Borehole U15n in Support of NCNS Source Physics Experiment. Report SAND2011-4394C, Sandia National Laboratories, Albuquerque, NM, 2011.

21. Broome S, Lee M. Triaxial compression testing results on core from borehole U15n, Nevada National Security Site, in support of NCNS Source Physics Experiment. Report SAND2013-2913P, , Sandia National Laboratories, Albuquerque, NM, 2013.

22. Sekine T, Duffy TS, Rubin AM, et al. Shock compression and isentropic release of granite. Geophys. J. Int. 1969;120:247-261. 


\section{Figure Captions}

Figure 1. Stress-strain responses: (a) uniaxial compression (experimental data obtained from [20]; (b) uniaxial tension (estimated responses).

Figure 2. Response under triaxial stress conditions for different confining stresses.

Figure 3. Granite response to strain controlled cyclic loading.

Figure 4. Uniaxial compressive strength as a function of normalized strain rate.

\section{Table Headings}

Table 1. Material properties for a Climax-Stock granite 
Table 1: Material properties for a Climax-Stock granite

\begin{tabular}{|l|c|}
\hline Material Properties & Values \\
\hline Density and Elastic Properties & $\rho=2630 \mathrm{~kg} / \mathrm{m}^{3}$ \\
& $B=46.22 \mathrm{GPa} ; \mu=31.4 \mathrm{GPa} ; \Gamma_{0}=1.5 ; S_{p}=0.6$ \\
\hline Initial Anisotropy & $\omega_{1}=\omega_{2}=\omega_{3}=1$ \\
\hline Strength & $\sigma_{0}^{S}=53.4 M P a ; q_{0}=1.4 ; \sigma_{q}=1.45 G P a ; n_{q}=3.8$ \\
\hline Dynamic Overstress & $\dot{e}_{N}^{c r}=500 \mathrm{sec}^{-1} ; \alpha_{D}=0.1$ \\
\hline Rate Dependence & $\omega_{p}=0.9 ; \dot{e}_{N}^{c r}=500.0 / \mathrm{s}$ \\
\hline Fracture & $G_{f}^{0}=6 \mathrm{KPa}_{2} n=4.0$ \\
& $W^{0}=150 \mathrm{kPa} ; m=1.2$ \\
\hline
\end{tabular}



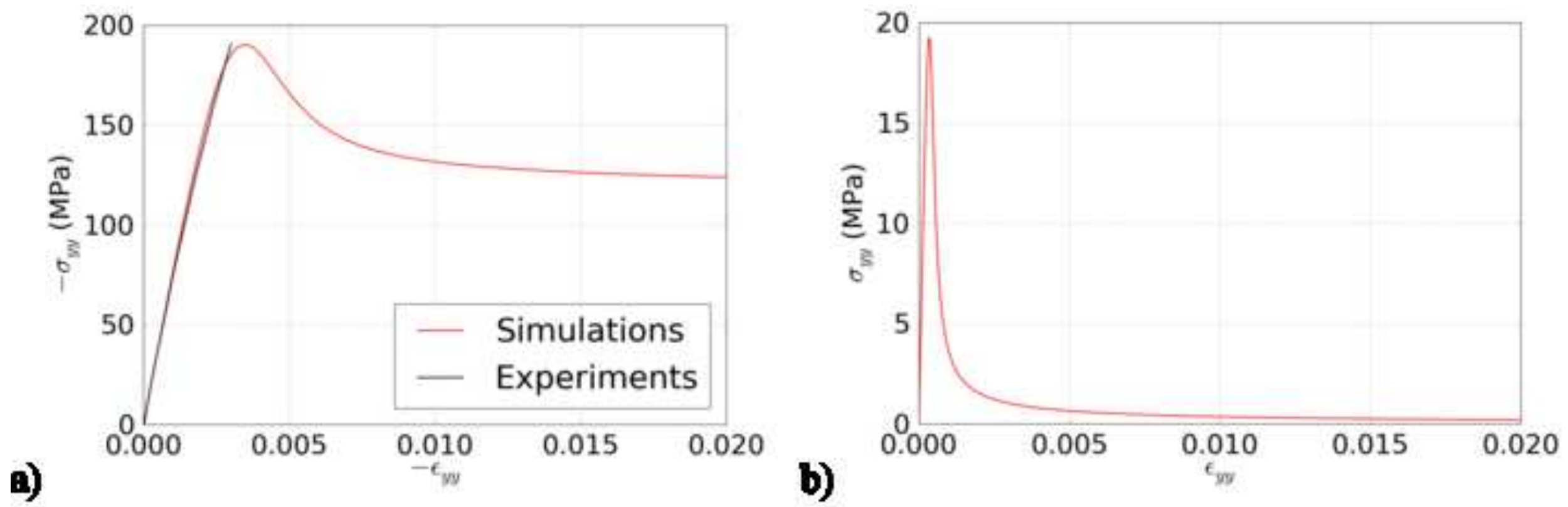


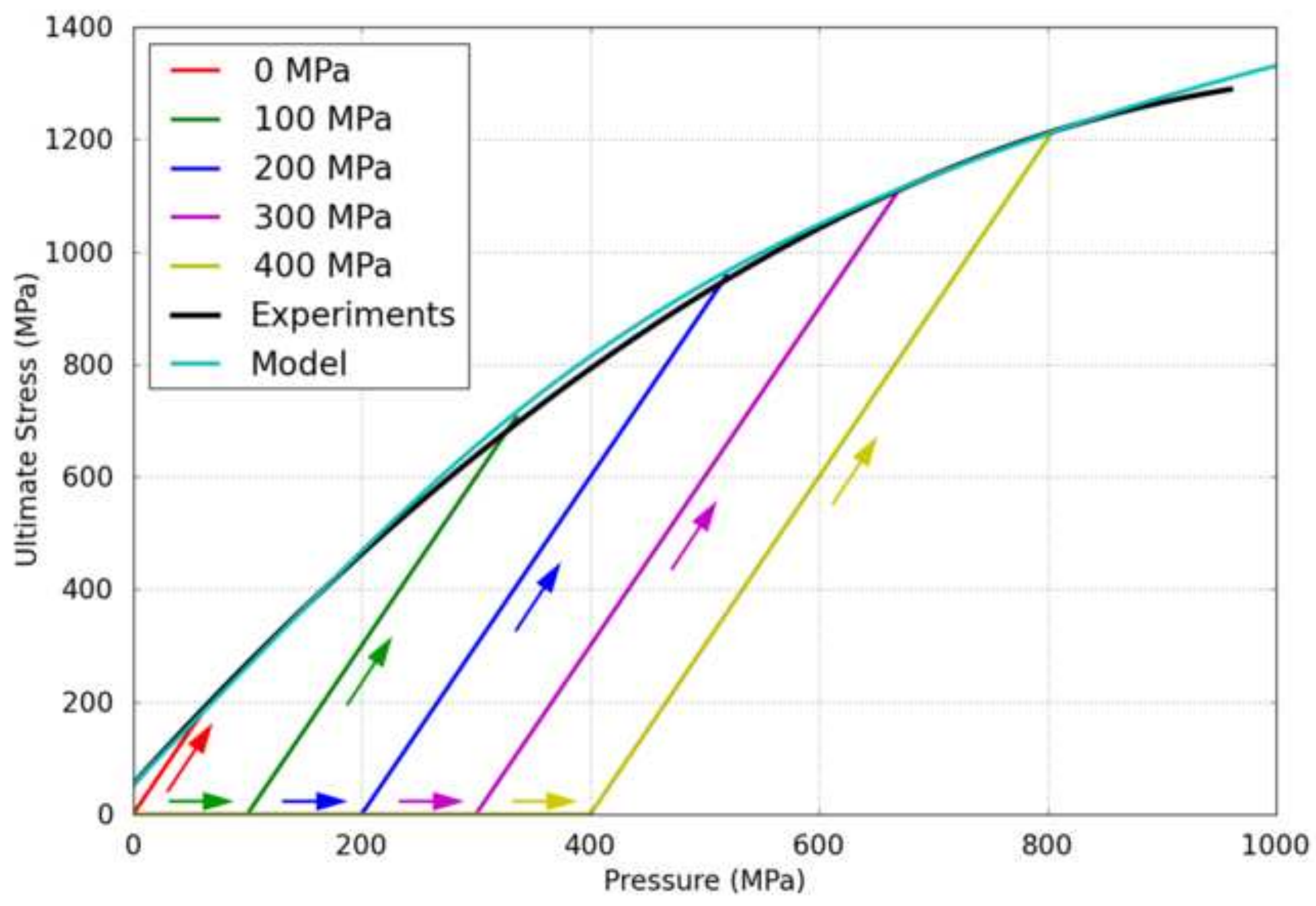




\section{Figure 3}

Click here to download high resolution image

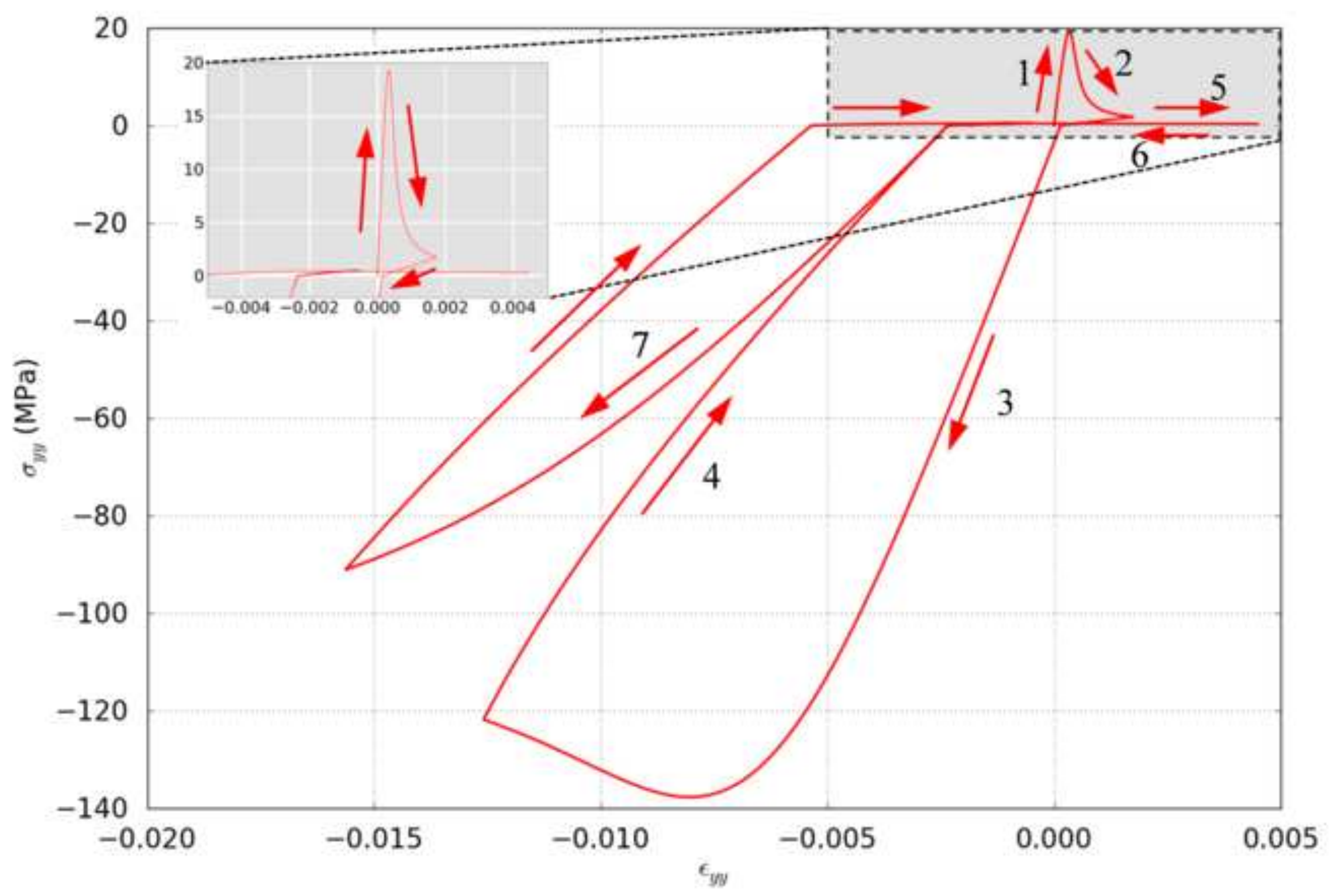


Click here to download high resolution image

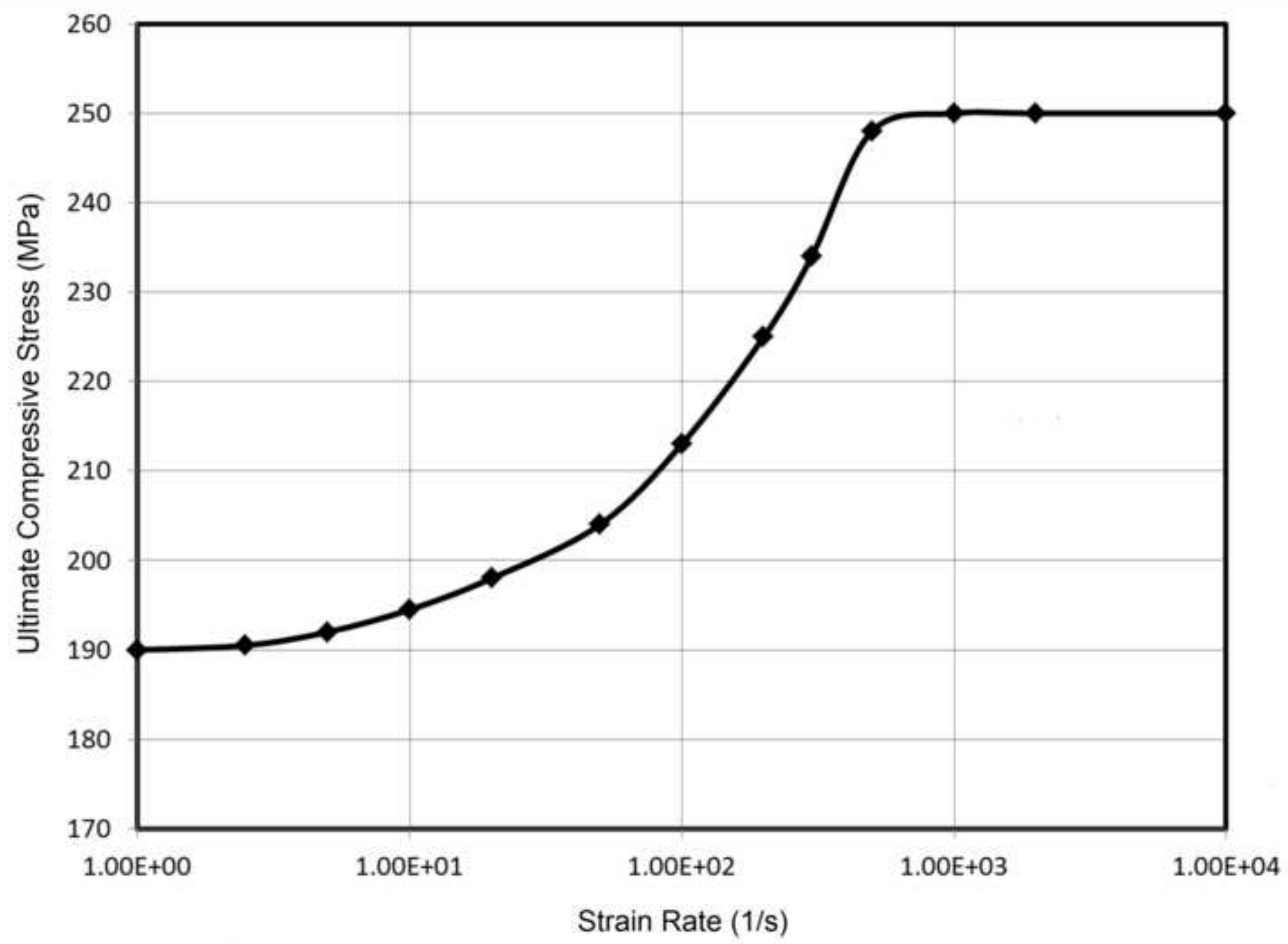

